\title{
Une généralisation du problème de Waring-Goldbach polynomial
}

\author{
par \\ Houda Aïssa (Tunis)
}

I. Introduction. Soit $\mathbb{F}_{q}$ le corps fini à $q$ éléments, $q$ étant impair, et $\mathbb{F}_{q}[X]$ l'anneau des polynômes à une variable sur le corps $\mathbb{F}_{q}$. Certaines analogies entre les propriétés arithmétiques de l'anneau $\mathbb{F}_{q}[X]$ et l'anneau $\mathbb{Z}$ des entiers relatifs ont été mises en évidence. En particulier, en ce qui concerne l'arithmétique additive, les problèmes de Waring [18] et de Goldbach [14] ont été étudiés et plus particulièrement le problème de Waring pour les carrés $([6]-[12])$.

Dans [2], M. Car a établi une estimation asymptotique du nombre $\varrho(s, M)$ de représentations d'un polynôme $M \in \mathbb{F}_{q}[X]$ comme somme de $s$ carrés de polynômes irréductibles :

$$
M=P_{1}^{2}+\ldots+P_{s}^{2},
$$

$P_{1}, \ldots, P_{s}$ étant des polynômes satisfaisant aux conditions de degré les plus restrictives possibles, à savoir pour tout $i=1, \ldots, s$,

$$
\operatorname{deg} P_{i} \leq n \quad \text { si } \operatorname{deg} M \in\{2 n, 2 n-1\},
$$

et cela pour $s \geq 5$.

D'autre part dans [5], M. Car a établi une estimation asymptotique du nombre $R\left(A_{1}, \ldots, A_{s} ; M\right)$ de représentations d'un polynôme $M \in \mathbb{F}_{q}[X]$ comme somme

$$
M=A_{1} Y_{1}^{2}+\ldots+A_{s} Y_{s}^{2},
$$

les polynômes $A_{1}, \ldots, A_{s}$ étant donnés premiers entre eux deux à deux, les polynômes $Y_{1}, \ldots, Y_{s}$ satisfaisant aux conditions de degré les plus restrictives possibles.

Une généralisation naturelle de ces deux problèmes est la suivante : étant donnés $A_{1}, \ldots, A_{s}$ polynômes de $\mathbb{F}_{q}[X]$, peut on représenter les polynômes $M \in \mathbb{F}_{q}[X]$ comme somme

$$
M=A_{1} P_{1}^{2}+\ldots+A_{s} P_{s}^{2},
$$

2000 Mathematics Subject Classification: 11P55, 11E25. 
$P_{1}, \ldots, P_{s}$ étant des polynômes irréductibles de $\mathbb{F}_{q}[X]$ satisfaisant aux conditions de degré les plus restrictives possibles, à savoir, pour $i=1, \ldots, s$,

$$
\operatorname{deg} P_{i} \leq m_{i} \text { ? }
$$

Ici $m_{i}$ est défini par

$$
\operatorname{deg} M-\operatorname{deg} A_{i}= \begin{cases}2 m_{i} & \text { si } \operatorname{deg} M-\operatorname{deg} A_{i} \text { est pair, } \\ 2 m_{i}-1 & \text { si } \operatorname{deg} M-\operatorname{deg} A_{i} \text { est impair. }\end{cases}
$$

Conformément à la terminologie de G. Effinger et D. Hayes [13], une représentation (1) satisfaisant la condition (2) est appelée une représentation stricte.

Comme le cas $\operatorname{deg} A_{1}=\ldots=\operatorname{deg} A_{s}=0$ est semblable au cas $A_{1}=$ $\ldots=A_{s}=1$ déjà étudié dans [2], on supposera qu'au moins un polynôme parmi $A_{1}, \ldots, A_{s}$ est non constant.

Comme il a déjà été noté dans [5], sans hypothèses supplémentaires sur les polynômes $A_{1}, \ldots, A_{s}$, il existe une infinité de polynômes $M \in \mathbb{F}_{q}[X]$ n'admettant pas de représentation (1). Dans ce qui suit, nous supposerons les polynômes $A_{1}, \ldots, A_{s}$ premiers entre eux deux à deux. Sous ces hypothèses nous obtenons le résultat suivant :

ThÉorème. Soient un entier $s \geq 5$ et $A_{1}, \ldots, A_{s}$ des polynômes de $\mathbb{F}_{q}[X]$ premiers entre eux deux à deux. Alors :

(i) Si $q=7$ ou $q>9$ tout polynôme $M \in \mathbb{F}_{q}[X]$ de degré assez grand admet une représentation stricte comme somme

$$
M=A_{1} P_{1}^{2}+\ldots+A_{s} P_{s}^{2} .
$$

De plus, si $R_{s}(M)$ désigne le nombre de ces représentations on a

$$
R_{s}(M) \gg(\operatorname{deg} M)^{-s}|M|^{s / 2-1},
$$

la constante intervenant dans le symbole $\gg$ ne dépendant que de $q$, s et $A_{1}, \ldots, A_{s}$.

(ii) Si $q=5$ ou $q=9$ et au moins quatre parmi $A_{1}, \ldots, A_{s}$ ont des degrés de même parité, soient $A_{i_{1}}, \ldots, A_{i_{4}}$, le résultat précédent reste valable pour les polynômes $M$ tel que $\operatorname{deg} M \equiv \operatorname{deg} A_{i_{1}} \bmod 2$.

La condition $s \geq 5$ est une condition technique. En fait, cette condition est nécessaire pour la convergence des séries singulières $\mathfrak{S}_{s}(M)$ (voir Proposition IV.9). Jusqu'à maintenant on n'a pas de démonstration pour le cas $s=4$ et on ne peut pas donner de contre-exemple.

II. Notations et conventions. Nous reprenons ici - en les rappelant - les notations utilisées dans [3] et [5]. Dans cet article, le mot polynôme désignera un polynôme de $\mathbb{F}_{q}[X]$. On désigne par $\mathbb{M}$ l'ensemble des polynômes unitaires, par $\mathbb{M}_{l}$ l'ensemble des polynômes unitaires de degré $l$, par 
$I$ l'ensemble des polynômes irréductibles unitaires et par $I^{*}$ l'ensemble des polynômes irréductibles quelconques. Si $A$ et $B$ sont des polynômes non nuls, on note $(A, B)$ leur pgcd unitaire.

Soit $H$ un polynôme non nul. On note $\operatorname{deg} H$ son degré, et $\mathcal{C}_{H}$ l'ensemble des polynômes de degré strictement inférieur au degré de $H$ identifié à l'ensemble des classes de congruence modulo $H$. Le groupe multiplicatif des classes inversibles modulo $H$ sera noté $\mathcal{C}_{H}^{*}$, l'ordre de ce groupe sera noté $\Phi(H)$. La fonction $\Phi$ ainsi définie a les mêmes propriétés que la fonction d'Euler classique. Sur le corps $\mathbb{K}=\mathbb{F}_{q}(X)$ des fractions rationnelles, on définit une valuation $v$ par

$$
v\left(\frac{A}{B}\right)=\operatorname{deg} B-\operatorname{deg} A
$$

si $A$ et $B$ sont des polynômes non nuls. Le complété $\mathbb{K}_{\infty}$ de $\mathbb{K}$ pour cette valuation s'identifie au corps $\mathbb{F}_{q}\left(\left(X^{-1}\right)\right)$ des séries de Laurent en $1 / X$ sur le corps $\mathbb{F}_{q}$, la valuation $v$ se prolongeant aux éléments non nuls de $\mathbb{K}_{\infty}$ par

$$
v\left(\sum_{s=-\infty}^{\infty} a_{s} X^{s}\right)=-\sup \left\{s \in \mathbb{Z} ; a_{s} \neq 0\right\} .
$$

On associe à cette valuation la valeur absolue ||$_{\infty}$ définie par

$$
|\alpha|_{\infty}= \begin{cases}q^{-v(\alpha)} & \text { si } \alpha \neq 0, \\ 0 & \text { si } \alpha=0 .\end{cases}
$$

Nous noterons simplement || cette valeur absolue car le contexte nous permet de la distinguer de la valeur absolue classique sur le corps $\mathbb{R}$ des nombres réels ou le corps $\mathbb{C}$ des nombres complexes qu'on va utiliser.

Soit $u \in \mathbb{K}_{\infty} ;$ si $u=\sum_{s=-\infty}^{\infty} u_{s} X^{s}$, on pose $\operatorname{Res}(u)=u_{-1}$. De plus, pour tout non nul $u \in \mathbb{K}_{\infty}$, on pose $\operatorname{sgn}(u)=u_{-v(u)}$.

Si $B$ est un sous-ensemble non vide de $\{1, \ldots, s\}$, on désigne par \#B le cardinal de $B$. D'autre part, si $\alpha_{1}, \ldots, \alpha_{s}$ sont des éléments de $\mathbb{F}_{q}$, pour tout $x \in \mathbb{F}_{q}$ on note $r_{B}\left(\alpha_{1}, \ldots, \alpha_{s} ; x\right)$ le nombre de solutions $\left(x_{i}\right)_{i \in B} \in\left(\mathbb{F}_{q}^{*}\right)^{\# B}$ de l'equation

$$
x=\sum_{i \in B} \alpha_{i} x_{i}^{2}
$$

Pour tout entier $j \geq 1$, on pose

$$
\tau_{j}=\sum_{k=1}^{j}(q-1) \frac{q^{k}}{k} .
$$

III. La méthode du cercle. Soit $\Psi$ le caractère additif défini sur $\mathbb{F}_{q}$ par

$$
\Psi(x)=\exp \left(\frac{2 i \pi \operatorname{tr}(x)}{p}\right),
$$


où tr est l'application trace de $\mathbb{F}_{q}$ dans $\mathbb{F}_{p}$. Au caractère non trivial $\Psi$, on associe le caractère additif non trivial $E$ de $\mathbb{K}_{\infty}$ défini par

$$
E(u)=\Psi(\operatorname{Res}(u)) .
$$

On désigne par $\mathcal{P}$ l'idéal de valuation, et, pour tout entier $j$, par $\mathcal{P}_{j}$ l'idéal

$$
\left\{t \in \mathbb{K}_{\infty} ; v(t)>j\right\} \text {. }
$$

Les ensembles $\mathcal{P}_{j}$ sont des sous-groupes compacts du groupe additif localement compact $\mathbb{K}_{\infty}$. Désignons par $d t$ la mesure de Haar sur $\mathbb{K}_{\infty}$ normalisée à $1 \operatorname{sur} \mathcal{P}$.

Nous rappelons ici quelques résultats établis dans [14] que nous utiliserons fréquemment par la suite. $q^{-j}$.

Proposition III.1. (i) Pour tout entier rationnel $j, \mathcal{P}_{j}$ a pour mesure

(ii) Pour tout $H \in \mathbb{F}_{q}[T], E(H)=1$.

(iii) Soit $H$ un polynôme non nul et soient $A$ et $B$ deux polynômes. Alors,

$$
A \equiv B(\bmod H) \Rightarrow E\left(\frac{A}{H}\right)=E\left(\frac{B}{H}\right) .
$$

(iv) Pour tout $u \in \mathbb{K}_{\infty}$, on a

$$
v(u) \geq 2 \Rightarrow E(u)=1 .
$$

(v) Soient un entier $j \geq 0, u \in \mathbb{K}_{\infty}$ et $b \in \mathcal{P}$. Alors,

$$
\int_{b+\mathcal{P}_{j}} E(u t) d t= \begin{cases}q^{-j} E(u b) & \text { si } v(u)>-j, \\ 0 & \text { si } v(u) \leq-j .\end{cases}
$$

(vi) Soient $G$ et $H$ des polynômes, $H$ n'étant pas nul. Alors,

$$
\sum_{R \in \mathcal{C}_{H}} E\left(\frac{G}{H} R\right)= \begin{cases}|H| & \text { si } H \text { divise } G, \\ 0 & \text { si } H \text { ne divise pas } G .\end{cases}
$$

Soit un entier $s \geq 5$. Soient $A_{1}, \ldots, A_{s}$ des polynômes premiers entre eux, l'un d'entre eux au moins étant non constant. Pour $i=1, \ldots, s$, soient

$$
a_{i}=\operatorname{deg} A_{i}, \quad \alpha_{i}=\operatorname{sgn}\left(A_{i}\right) .
$$

Sans perte de généralité on peut supposer que $a_{1} \leq \ldots \leq a_{s}$. On a donc $a_{s} \neq 0$. Soit $n$ un entier naturel tel que

$$
\begin{aligned}
n^{1 / 3} \geq & 2\left(2 q(\log q)^{-2}+\frac{1}{4}\right)+\frac{a_{s}}{2}+\frac{1}{2}, \\
& n-4 n^{1 / 3} \geq \frac{3\left(a_{s}+1\right)}{2} .
\end{aligned}
$$

Soit $M$ un polynôme de degré $2 n$ ou $2 n-1$. Soit $R_{s}\left(A_{1}, \ldots, A_{s} ; M\right)$ le nombre de solutions $\left(P_{1}, \ldots, P_{s}\right) \in\left(\mathbb{F}_{q}[X]\right)^{s}$ de l'equation (1), vérifiant les conditions de degré (2). 
On rappelle que pour tout $i=1, \ldots, s$,

$$
\operatorname{deg} M-\operatorname{deg} A_{i}= \begin{cases}2 m_{i} & \text { si } \operatorname{deg} M-\operatorname{deg} A_{i} \text { est pair, } \\ 2 m_{i}-1 & \text { si } \operatorname{deg} M-\operatorname{deg} A_{i} \text { est impair. }\end{cases}
$$

Soit $u(M)$, respectivement $v(M)$, l'ensemble des $i \in\{1, \ldots, s\}$ tel que $\operatorname{deg} M \equiv a_{i} \bmod 2$, respectivement $\operatorname{deg} M \not \equiv a_{i} \bmod 2$. Pour $i=1, \ldots, s$, on note $f_{i}$ l'application de $\mathcal{P}$ dans $\mathbb{C}$ définie par

$$
f_{i}(t)=\sum_{\substack{P \in I^{*} \\ \operatorname{deg} P \leq m_{i}}} E\left(t A_{i} P^{2}\right) .
$$

On pose

$$
F(t)=\prod_{i=1}^{s} f_{i}(t) .
$$

Alors, d'après la relation (III.1) on a

$$
R_{s}\left(A_{1}, \ldots, A_{s} ; M\right)=\int_{\mathcal{P}} F(t) E(-M t) d t .
$$

Pour alléger les notations, quand il n'y aura pas d'ambiguité, nous noterons

$$
R_{s}\left(A_{1}, \ldots, A_{s} ; M\right)=R_{s}(M) .
$$

DÉfinition 1. Soit un entier $l \geq 0$. On appelle fraction de Farey à l'ordre $l$ toute fraction rationnelle $G / H$ telle que :

(i) $H$ est un polynôme unitaire de degré $\leq l$,

(ii) $G$ et $H$ sont des polynômes premiers entre eux,

(iii) $\operatorname{deg} G<\operatorname{deg} H$.

Si $G / H$ est une fraction de Farey à l'ordre $l$, on appelle arc de Farey à l'ordre $l$ de centre $G / H$ la boule

$$
\mathcal{U}_{G / H, l}=\{t \in \mathcal{P} ; v(t-G / H)>l+\operatorname{deg} H\} .
$$

On désigne par $\mathcal{F}_{l}$ l'ensemble des fractions de Farey à l'ordre $l$. On a alors :

Proposition III.2 ([14]). Soit un entier $l \geq 0$. Lorsque $G / H$ décrit $\mathcal{F}_{l}$, les arcs de Farey $\mathcal{U}_{G / H, l}$ forment une partition de $\mathcal{P}$.

Une telle partition est appelée la dissection de Farey à l'ordre $l$.

Dans ce qui suit nous utiliserons deux dissections de Farey. La deuxième sera définie au chapitre VI. La première est une dissection de Farey à l'ordre

$$
N=2 n-4 r,
$$

où

$$
r=\left[n^{1 / 3}\right] .
$$

Nous noterons $\mathcal{U}_{G / H}$ l'arc de Farey $\mathcal{U}_{G / H, N}$. Les arcs de Farey $\mathcal{U}_{G / H}$ tels que $\operatorname{deg} H \leq 4 r$ seront dits majeurs. Soit $\mathcal{M}$ leur réunion. Les arcs restants 
sont répartis en deux catégories définies comme suit : Posons, pour tout $i=1, \ldots, s$,

$$
H_{i}^{*}=\frac{H}{\left(H, A_{i}\right)}, \quad A_{i}^{*}=\frac{A_{i}}{\left(H, A_{i}\right)} .
$$

Les arcs de Farey de centre $G / H$ où $\operatorname{deg} H>4 r$ et où pour tout $i=1, \ldots, s$ $\operatorname{deg} H_{i}^{*} \leq 4 r$ seront dits arcs médians. Notons $\mathcal{M}_{1}^{\prime}$ leur réunion. Sur les arcs majeurs comme sur les arcs médians, on a une bonne approximation des fonctions $f_{i}(t)$. Enfin les arcs de Farey de centre $G / H$ pour lesquels existe $i \in\{1, \ldots, s\}$ tel que $\operatorname{deg} H_{i}^{*}>4 r$ seront dits arcs mineurs. Notons $\mathcal{M}_{2}^{\prime}$ leur réunion.

Soit

$$
I_{G / H}(M)=\int_{\mathcal{U}_{G / H}} F(t) E(-M t) d t
$$

La somme

$$
\int_{\mathcal{M}} F(t) E(-M t) d t=\sum_{\substack{H \in \mathbb{M} \\ \operatorname{deg} H \leq 4 r}} \sum_{G \in \mathcal{C}_{H}^{*}} I_{G / H}(M)
$$

donnera de bonne approximation de $R_{s}(M)$. Le calcul de cette somme fait apparaître les premiers termes d'une série singulière $\mathfrak{S}_{s}(M)$ qui sera étudiée au paragraphe suivant.

Posons

$$
\begin{aligned}
& R^{+}(M)=\int_{\mathcal{M}} F(t) E(-M t) d t, \\
& R_{1}^{-}(M)=\int_{\mathcal{M}_{1}^{\prime}} F(t) E(-M t) d t, \\
& R_{2}^{-}(M)=\int_{\mathcal{M}_{2}^{\prime}} F(t) E(-M t) d t .
\end{aligned}
$$

Convenons que, sans indications supplémentaires, les constantes contenues dans les symboles $\ll$ et $\gg$ ne dépendront que de $q, s$ et $A_{1}, \ldots, A_{s}$ ou seront absolues.

IV. Les séries singulières. Dans ce paragraphe, $M$ est un polynôme fixé. On pose, pour tout polynôme unitaire $H$ et pour $G$ premier à $H$

$$
\begin{aligned}
& S(H, G)=\sum_{R \in \mathcal{C}_{H}^{*}} E\left(\frac{G}{H} R^{2}\right), \\
& T(H, G)=\prod_{i=1}^{s} S\left(H, G A_{i}\right) .
\end{aligned}
$$


Si $H$ est un polynôme non nul, on pose

$$
A_{s}(H, M)=\Phi(H)^{-s} \sum_{G \in \mathcal{C}_{H}^{*}} T(H, G) E\left(-M \frac{G}{H}\right) .
$$

Proposition IV.1. Soient $P_{1}, \ldots, P_{r}$ des polynômes irréductibles unitaires et $k_{1}, \ldots, k_{r}$ des entiers naturels. Alors, pour tout polynôme $A$, on a

$$
S\left(\prod_{i=1}^{r} P_{i}^{k_{i}}, A\right)=\prod_{i=1}^{r} S\left(P_{i}^{k_{i}}, A \prod_{j=1, j \neq i}^{r} P_{j}^{k_{j}}\right) .
$$

Démonstration. C'est la proposition III.2 de [5].

Proposition IV.2. Soient $P$ un polynôme irréductible, $G$ un polynôme premier à $P$. Alors on $a$ :

(i) Pour tout entier $l \geq 2$,

$$
S\left(P^{l}, G\right)=0 .
$$

$$
|S(P, G)| \leq 1+|P|^{1 / 2} .
$$

(iii) Si $B$ est un polynôme non $n u l, v=v_{P}(B), B^{*}=B / P^{v}$ et si $k$ est un entier $\geq 0$,

$$
S\left(P^{k}, B\right)= \begin{cases}\Phi(P)^{k} & \text { si } k \leq v \\ |P|^{v} S\left(P^{k-v}, B^{*}\right) & \text { si } k>v .\end{cases}
$$

Démonstration. (i) et (ii) sont les propositions V.2 et V.3 de [2], et (iii) se démontre comme (iii) de la proposition de [5].

Lemme 1 ([4, lemme, p. 14]). Pour tout réel $\theta>1$, pour tout réel $\varepsilon>0$, il existe une constante $a=a(q, \theta, \varepsilon)$ telle que, pour tout polynôme $H$ sans facteur carré, on ait

$$
\theta^{\omega(H)} \leq a(q, \theta, \varepsilon)|H|^{\varepsilon},
$$

où $\omega(H)$ est le nombre de facteurs irréductibles de $H$.

Corollaire 1. Soit $\varepsilon$ un réel positif. Soient $H$ un polynôme unitaire et $A$ un polynôme quelconque. Alors, pour tout polynôme $G$ premier à $H$, on a

$$
|S(H, G A)| \ll|H|^{1 / 2+\varepsilon},
$$

où la constante intervenant dans le symbole «ne dépend que de q, $\varepsilon$ et $A$.

Démonstration. Le résultat se déduit à l'aide des relations (IV.4)-(IV.8).

Proposition IV.3. La fonction $H \mapsto A_{s}(H, M)$ est multiplicative.

Démonstration. Immédiate. 
Proposition IV.4. Soit $H$ un polynôme avec facteur carré. Alors (IV.10)

$$
A_{s}(H, M)=0 .
$$

Démonstration. Soit $P$ un polynôme irréductible tel que $P^{2}$ divise $H$. Puisque $A_{1}, \ldots, A_{s}$ sont premiers entre eux, il existe $i \in\{1, \ldots, s\}$ tel que $P$ ne divise pas $A_{i}$. D'après les relations (IV.2) et (IV.5) on a $T\left(P^{v_{P}(H)}, G\right)=0$, d'où avec (IV.3), $A_{s}\left(P^{v_{P}(H)}, M\right)=0$ et par multiplicativité $A_{s}(H, M)=0$.

Pour tout polynôme irréductible $P$, on pose

$$
\Psi_{s}(P, M)=1+A_{s}(P, M) .
$$

On désigne par $\mathcal{N}_{s}(P, M)$ le nombre de solutions $\left(M_{1}, \ldots, M_{s}\right)$ de la congruence

$$
M \equiv A_{1} M_{1}^{2}+\ldots+A_{s} M_{s}^{2}(\bmod P)
$$

telles que $M_{1}, \ldots, M_{s}$ soient non nuls modulo $P$.

Proposition IV.5. Soit $P$ un polynôme irréductible. Alors on a

$$
\Phi(P)^{s} \Psi_{s}(P, M)=|P| \mathcal{N}_{s}(P, M) .
$$

Démonstration. D’après (IV.1)-(IV.3) et (III.2),

$$
\begin{aligned}
\Phi(P)^{s} A_{s}(P, M) & =\sum_{G \in \mathcal{C}_{P}^{*}} \prod_{i=1}^{s}\left(\sum_{R \in \mathcal{C}_{P}^{*}} E\left(\frac{G A_{i}}{P} R^{2}\right)\right) E\left(-M \frac{G}{P}\right) \\
& =\sum_{R_{1}, \ldots, R_{s} \in \mathcal{C}_{P}^{*}} \sum_{G \in \mathcal{C}_{P}^{*}} E\left(\frac{G}{P}\left(A_{1} R_{1}^{2}+\ldots+A_{s} R_{s}^{2}-M\right)\right) \\
& =\sum_{R_{1}, \ldots, R_{s} \in \mathcal{C}_{P}^{*}}\left(\sum_{G \in \mathcal{C}_{P}}\left(\frac{G}{P}\left(A_{1} R_{1}^{2}+\ldots+A_{s} R_{s}^{2}-M\right)\right)-1\right) \\
& =|P| \mathcal{N}_{s}(P, M)-\Phi(P)^{s} .
\end{aligned}
$$

L'étude des congruences modulo un polynôme irréductible $P$ revient à étudier des équations dans un corps fini à $|P|$ éléments. Le résultat dont on a besoin est donné par la proposition suivante :

Proposition IV.6. Soit un entier $m \geq 3$. Soient $b_{1}, \ldots, b_{m}$ des éléments non nuls du corps $\mathbb{F}_{q}$. Pour tout $b \in \mathbb{F}_{q}$, soit

$$
r_{m}^{*}(b)=r^{*}\left(b_{1}, \ldots, b_{m} ; b\right)
$$

le nombre de solutions $\left(x_{1}, \ldots, x_{m}\right) \in\left(\mathbb{F}_{q}^{*}\right)^{m}$ de l'équation

$$
b=b_{1} x_{1}^{2}+\ldots+b_{m} x_{m}^{2} .
$$

Alors, on a

$$
\left|q r_{m}^{*}(b)-(q-1)^{m}\right| \leq(q-1)(1+\sqrt{q})^{m} .
$$


Démonstration. Pour $t \in \mathbb{F}_{q}$, posons

$$
\sigma^{*}(t)=\sum_{x \in \mathbb{F}_{q}^{*}} \Psi\left(t x^{2}\right)
$$

Alors on a

$$
q r_{m}^{*}(b)=\sum_{t \in \mathbb{F}_{q}} \Psi(-t b) \prod_{i=1}^{m} \sigma^{*}\left(t b_{i}\right)
$$

d'où

$$
q r_{m}^{*}(b)=\sigma^{*}(0)^{m}+\sum_{t \in \mathbb{F}_{q}^{*}} \Psi(-t b) \prod_{i=1}^{m} \sigma^{*}\left(t b_{i}\right) .
$$

On déduit de la relation (5.9) de [16] que $\left|\sigma^{*}\left(t b_{i}\right)\right| \leq 1+\sqrt{q}$ pour tout $t$ non nul, ce qui donne

$$
\left|q r_{m}^{*}(b)-(q-1)^{m}\right| \leq(q-1)(1+\sqrt{q})^{m} .
$$

Proposition IV.7. (i) Pour $q \geq 5$ et $m \geq 4$, et tout $b \in \mathbb{F}_{q}$, on $a$

$$
r_{m}^{*}(b)>0 .
$$

(ii) Pour $q=7$ et $q>9$, et tout $b \in \mathbb{F}_{q}$, on $a$

$$
r_{3}^{*}(b)>0 .
$$

Démonstration. (i) D'après (IV.13)

$$
q r_{m}^{*}(b) \geq(q-1)^{m}-(q-1)(1+\sqrt{q})^{m} .
$$

Si $q \geq 7$ et $m \geq 4$, le membre droit est $>0$. Si $q=5$, on montre directement que $r_{4}^{*}(b)>0$, et par suite $r_{m}^{*}(b)>0$ pour tout $m \geq 4$.

(ii) Si $q>9$, on déduit de (IV.13) que $r_{3}^{*}(b)>0$. Si $q=7$, on le démontre directement.

Corollaire 2. Soit s un entier supérieur ou égal à 5 et $P$ un polynôme irréductible. Alors,

$$
\Psi_{s}(P, M) \geq \Phi(P)^{-s}\left((|P|-1)^{s-1}-(|P|-1)\left(1+|P|^{1 / 2}\right)^{s-1}\right) .
$$

Démonstration. On remarque que si $P$ ne divise aucun des polynômes $A_{1}, \ldots, A_{s}$ alors

$$
\mathcal{N}_{s}(P, M)=r_{s}^{*}\left(\bar{A}_{1}, \ldots, \bar{A}_{s} ; \bar{M}\right),
$$

où pour tout polynôme $Y$ de $\mathbb{F}_{q}[X], \bar{Y}$ désigne la classe de $Y$ modulo $P$. Si $P$ divise l'un des polynômes $A_{1}, \ldots, A_{s}$, soit par exemple $A_{s}$, on a

$$
\mathcal{N}_{s}(P, M)=r_{s-1}^{*}\left(\bar{A}_{1}, \ldots, \bar{A}_{s-1} ; \bar{M}\right) .
$$

La relation (IV.16) se déduit alors de (IV.13). 
Proposition IV.8. Soit $P$ un polynôme irréductible. Alors on a

$$
\left|A_{s}(P, M)\right| \leq\left(\frac{2\left|A_{s}\right|^{1 / 2} q}{q-1}\right)^{s}|P|^{1-s / 2} .
$$

Démonstration. Conséquence des relations (IV.3), (IV.6) et (IV.7).

Proposition IV.9. (i) Pour $s \geq 5$, la série

$$
\mathfrak{S}_{s}(M)=\sum_{H \in \mathbb{M}} A_{s}(H, M)
$$

est absolument convergente, et on a

$$
\mathfrak{S}_{s}(M)=\prod_{P \in I} \Psi_{s}(P, M) .
$$

De plus, pour tout entier $t \geq 0$ et tout $\varepsilon \in] 0,1 / 2[$, on a

$$
\sum_{\substack{H \in \mathbb{M} \\ \operatorname{deg} H>t}}\left|A_{s}(H, M)\right| \ll q^{t(\varepsilon+2-s / 2)},
$$

la constante impliquée par le symbole « ne dépendant que de $q, s, \varepsilon, A_{1}, \ldots$ $\ldots, A_{s}$.

(ii) Il existe des constantes $a_{1}=a_{1}\left(q, s, A_{1}, \ldots, A_{s}\right), a_{2}=a_{2}\left(q, s, A_{1}, \ldots\right.$ $\left.\ldots, A_{s}\right)$, strictement positives, telles que

$$
a_{1} \leq \mathfrak{S}_{s}(M) \leq a_{2} .
$$

Démonstration. Soit $H$ un polynôme sans facteur carré. D'après (IV.17),

(*) $\left|A_{s}(H, M)\right| \leq \Phi(H)^{-s} \sum_{G \in \mathcal{C}_{H}^{*}}|T(H, G)| \leq\left(\frac{2\left|A_{s}\right|^{1 / 2} q}{q-1}\right)^{s \omega(H)}|H|^{1-s / 2}$.

D'après le lemme précédent, pour tout nombre réel $\varepsilon>0$,

$$
\left|A_{s}(H, M)\right| \leq \Phi(H)^{-s} \sum_{G \in \mathcal{C}_{H}^{*}}|T(H, G)| \ll|H|^{\varepsilon+1-s / 2},
$$

la constante impliquée par $\ll$ ne dépendant que de $q, s, \varepsilon, A_{1}, \ldots, A_{s}$. La relation (IV.20) s'en déduit. Ceci prouve que la série $\mathfrak{S}_{s}(M)$ est absolument convergente. La fonction $H \mapsto A_{s}(M, H)$ étant multiplicative, la somme $\mathfrak{S}_{s}(M)$ s'écrit comme produit eulérien absolument convergent, d'où (IV.19).

Les relations (IV.11) et (IV.17) nous donnent

$$
1-\left(\frac{2\left|A_{s}\right|^{1 / 2} q}{q-1}\right)^{s}|P|^{1-s / 2} \leq \Psi_{s}(P, M) \leq 1+\left(\frac{2\left|A_{s}\right|^{1 / 2} q}{q-1}\right)^{s}|P|^{1-s / 2} .
$$

Le produit

$$
\prod_{P \in I}\left(1+\left(\frac{2\left|A_{s}\right|^{1 / 2} q}{q-1}\right)^{s}|P|^{1-s / 2}\right)
$$


est convergent. Soit $a_{2}$ ce produit. On a ainsi la deuxième inégalité de (IV.21).

Soit

$$
d=\frac{2 s \log \left(\frac{2\left|A_{s}\right|^{1 / 2} q}{q-1}\right)}{(s-2) \log q} .
$$

Si $P$ est un polynôme irréductible tel que $\operatorname{deg} P>d$, on a $\Psi_{s}(P, M)>0$. Le produit

$$
\gamma_{s}\left(q, A_{s}\right)=\prod_{P \in I, \operatorname{deg} P>d}\left(1-\left(\frac{2\left|A_{s}\right|^{1 / 2} q}{q-1}\right)^{s}|P|^{1-s / 2}\right)
$$

est convergent et strictement positif. On a

$$
\mathfrak{S}_{s}(M) \geq \gamma_{s}\left(q, A_{s}\right) \prod_{P \in I, \operatorname{deg} P \leq d} \Psi_{s}(P, M) .
$$

On pose

$$
a_{1}=\gamma_{s}\left(q, A_{s}\right) \prod_{P \in I, \operatorname{deg} P \leq d} \Phi(P)^{-s}\left((|P|-1)^{s-1}-(|P|-1)\left(1+|P|^{1 / 2}\right)^{s-1}\right)
$$

et la relation (IV.16) nous donne alors la première inégalité de (IV.21).

V. Estimation de $f_{i}(t)$. On peut déduire l'estimation de $f_{i}(t)$ de l'estimation de $f(t)$ définie par

$$
f(t)=\sum_{P \in I, \operatorname{deg} P \leq m} E\left(t A P^{2}\right),
$$

où $m$ est un entier non nul et $A$ un polynôme non nul.

Les théorèmes de répartition des nombres premiers dans les progressions arithmétiques se généralisent aux polynômes de $\mathbb{F}_{q}[X]$. On a les théorèmes suivants établis dans [15].

ThÉORÈme 1. Soit, pour tout entier $k>0, \pi(k)$ le nombre de polynômes irréductibles unitaires de degré $k$ de $\mathbb{F}_{q}[X]$. Alors,

$$
q^{k}-2 q^{k / 2} \leq k \pi(k) \leq q^{k} .
$$

ThÉORÈme 2. Soit, pour tout entier $k>0$, tout polynôme unitaire $H \neq 1$ et tout polynôme unitaire $R$ premier à $H, \Pi(k, H, R)$ le nombre de polynômes unitaires irréductibles de degré $k$ de $\mathbb{F}_{q}[X]$ congrus à $R$ modulo H. Alors,

$$
\left|\Pi(k, H, R)-\frac{q^{k}}{k \Phi(H)}\right| \leq \operatorname{deg} H \frac{q^{k / 2}}{k} .
$$

THÉORÈme 3. Soit, pour tout entier $k>0$, tout polynôme unitaire $H$, tout entier $l \geq 0$ tel que $l+\operatorname{deg} H \geq 1$, tout polynôme unitaire $R$ premier 
à $H, \Pi(k, H, l, R)$ le nombre de polynômes unitaires irréductibles $P$ de $\mathbb{F}_{q}[X]$ de degré $k$, congrus à $R$ modulo $H$ et tels que

$$
\operatorname{deg}\left(X^{\operatorname{deg} P} R-X^{\operatorname{deg} R} P\right)<\operatorname{deg} P+\operatorname{deg} R-l .
$$

Alors,

$$
\left|\Pi(k, H, l, R)-\frac{q^{k-l}}{k \Phi(H)}\right| \leq(l+\operatorname{deg} H+1) \frac{q^{k / 2}}{k} .
$$

Ce dernier théorème correspond à une partition des polynômes unitaires irréductibles suivant les différents restes modulo $H$, et les différents systèmes $\left(a_{k-1}, \ldots, a_{k-l}\right)$ possibles, pour les coefficients des $l$ termes de plus haut degré.

Lemme 2. Soit, pour tout polynôme $A$ non nul, $D(A)$ le nombre de diviseurs unitaires de A. Alors, pour tout $\delta>0$ il existe une constante $c_{1}=c_{1}(\delta)$ telle que

$$
D(A) \leq c_{1}|A|^{\delta} .
$$

Démonstration. Semblable à celle du lemme V.1, chap. IV de [1].

Rappelons ici que l'on a divisé l'ideal $\mathcal{P}$ par une dissection de Farey à l'ordre $N=2 n-4 r$, les entiers $n$ et $r$ vérifiant les relations (III.3), (III.4) et (III.5). Soit $A$ un polynôme non nul et soit $a$ son degré.

Proposition V.1. Soit $h$ un entier $<m$. Soit $t=u+G / H$ appartenant à un arc de Farey de centre $G / H$ où $\operatorname{deg} H<h$. Alors,

$$
\left|f(t)-\frac{S(H, G A)}{\Phi(H)} \sum_{k=1}^{m} \frac{q^{k}}{k} \Gamma_{k}^{\operatorname{sgn}(A)}(u)\right| \ll r|H| \frac{q^{5 m / 2-v(u)}}{m}+\frac{q^{h}}{h},
$$

où pour tout $\alpha \in \mathbb{F}_{q}^{*}$,

$$
\Gamma_{k}^{\alpha}(u)= \begin{cases}1 & \text { si } v(u)>a+2 k+1, \\ \Psi(\alpha \operatorname{sgn}(u)) & \text { si } v(u)=a+2 k+1, \\ 0 & \text { si } v(u) \leq a+2 k\end{cases}
$$

la constante intervenant dans le symbole $\ll$ ne dépendant que de q et $A$.

Démonstration. Dans ce qui suit, les constantes impliquées dans $\ll$ ne dépendront que de $q$ et de $A$.

On a

$$
f(t)=\sum_{\substack{P \in I \\ \operatorname{deg} P<h}} E\left(t A P^{2}\right)+\sum_{\substack{P \in I \\ h \leq \operatorname{deg} P \leq m}} E\left(t A P^{2}\right) .
$$

Posons

$$
f^{\prime}(t)=\sum_{\substack{P \in I \\ h \leq \operatorname{deg} P \leq m}} E\left(t A P^{2}\right)
$$


Alors,

$$
\left|f(t)-f^{\prime}(t)\right| \ll q^{h} / h,
$$

où la constante intervenant dans $\ll$ ne dépend que de $q$. On a

$$
f^{\prime}(t)=\sum_{k=h}^{m} \sum_{\substack{P \in I \\ \operatorname{deg} P=k}} E\left(t A P^{2}\right)=\sum_{k=h}^{m} g_{k}(t) .
$$

Soit $k \in\{h, \ldots, m\}$. Soit $l=l(k)=2 k-v(u)+a+1$. Sur l'ensemble des polynômes unitaires $\mathbb{M}$, on définit la relation d'equivalence $R_{H, l}$ comme suit : Pour tout $Y, Z \in \mathbb{M}$,

$$
Y \equiv Z \bmod R_{H, l} \Leftrightarrow\left\{\begin{array}{l}
\text { (i) } Y \equiv Z \bmod H \\
\text { (ii) } \operatorname{deg}\left(Y X^{\operatorname{deg} Z}-Z X^{\operatorname{deg} Y}\right)<\operatorname{deg}(Y Z)-l .
\end{array}\right.
$$

D'après la proposition III.1, si $\operatorname{deg} Y=\operatorname{deg} Z=k$ alors

$$
Y \equiv Z \bmod R_{H, l} \Rightarrow E\left(t A Y^{2}\right)=E\left(t A Z^{2}\right) .
$$

Si $l \leq 0$, les classes modulo $R_{H, l}$ sont les classes de congruences modulo $H$. Pour $l>0$, soit $\mathcal{S}=\left\{R+T^{w} H Y ; R \in \mathcal{C}_{H}, Y \in \mathbb{M}_{l}, w=k-l-\operatorname{deg} H\right\}$. D'après [4, proposition IX.1], $\mathcal{S}$ est un système de représentants de l'ensemble des polynômes unitaires irréductibles de degré $k$ modulo $R_{H, l}$.

Supposons $l \leq 0$, i.e. $v(u) \geq 2 k+a+1$. Comme $\operatorname{deg} H<h \leq \operatorname{deg} P$ alors $(P, H)=1$. On a

$$
\begin{aligned}
g_{k}(t) & =\sum_{R \in \mathcal{C}_{H}^{*}} \sum_{\substack{P \in I, \operatorname{deg} P=k \\
P \equiv R \bmod H}} E\left(\left(u+\frac{G}{H}\right) A P^{2}\right) \\
& =\sum_{R \in \mathcal{C}_{H}^{*}} E\left(\frac{G}{H} A R^{2}\right) \sum_{\substack{P \in I, \operatorname{deg} P=k \\
P \equiv R \bmod H}} E\left(u A P^{2}\right) .
\end{aligned}
$$

Pour $v(u)>2 k+a+1$,

$$
g_{k}(t)=\sum_{R \in \mathcal{C}_{H}^{*}} E\left(\frac{G}{H} A R^{2}\right) \Pi(k, H, R) .
$$

Pour $v(u)=2 k+a+1$,

$$
g_{k}(t)=\Psi(\operatorname{sgn}(u A)) \sum_{R \in \mathcal{C}_{H}^{*}} E\left(\frac{G}{H} A R^{2}\right) \Pi(k, H, R) .
$$

Supposons $l>0$, i.e. $v(u)<2 k+a+1$. D'après l'implication (2), on a

$$
g_{k}(t)=\sum_{\substack{K \in \mathcal{S} \\(H, K)=1}} E\left(t A K^{2}\right) \Pi(k, H, l, K) .
$$


Le théorème 3 donne alors

$$
\begin{aligned}
\left|g_{k}(t)-\frac{q^{k-l}}{k \Phi(H)} \sum_{\substack{K \in \mathcal{S} \\
(H, K)=1}} E\left(t A K^{2}\right)\right| & \leq(l+\operatorname{deg} H+1) \frac{q^{k / 2}}{k} \sum_{\substack{K \in \mathcal{S} \\
(H, K)=1}} 1 \\
& \leq(l+\operatorname{deg} H+1) \Phi(H) q^{l} \frac{q^{k / 2}}{k} .
\end{aligned}
$$

Calculons maintenant

$$
\sum_{\substack{K \in \mathcal{S} \\(H, K)=1}} E\left(t A K^{2}\right)=\sum_{R \in \mathcal{C}_{H}^{*}} E\left(\frac{G}{H} A R^{2}\right) \sum_{Y \in \mathbb{M}_{l}} E\left(u A\left(X^{w} H Y\right)^{2}\right) .
$$

Soit $Y \in \mathbb{M}_{l}, y \in \mathcal{P}$. On a

$$
v\left(u A\left(X^{w} H\right)^{2}\left(Y^{2}-(Y+y)^{2}\right)\right) \geq 2,
$$

d'où

$$
\begin{aligned}
\sum_{Y \in \mathbb{M}_{l}} E\left(u A\left(X^{w} H Y\right)^{2}\right) & =\sum_{Y \in \mathbb{M}_{l}} \int_{\mathcal{P}} E\left(u A\left(X^{w} H\right)^{2}(Y+y)^{2}\right) d y \\
& =\int_{\substack{\operatorname{sgn}(y)=1 \\
v(y)=-l}} E\left(u A\left(X^{w} H y\right)^{2}\right) d y .
\end{aligned}
$$

Soit $y$ tel que $\operatorname{sgn}(y)=1$ et $v(y)=-l$. Alors $y^{2}=T^{2 l}+z$ avec $v(z)>-2 l$. Ainsi,

$q^{l} \sum_{Y \in \mathbb{M}_{l}} E\left(u A\left(X^{w} H Y\right)^{2}\right)=E\left(u A\left(X^{w} H X^{l}\right)^{2}\right) \int_{v(z)>-2 l} E\left(u A X^{2 w} H^{2} z\right) d z=0$,

puisque $v\left(u A X^{2 w} H^{2}\right) \leq 2 l$. Avec le théorème 2 , on conclut que

$$
\begin{aligned}
\mid f^{\prime}(t) & -\frac{S(H, G A)}{\Phi(H)} \sum_{k=h}^{m} \frac{q^{k}}{k} \Gamma_{k}^{\operatorname{sgn}(A)}(u) \mid \\
& \leq \operatorname{deg} H|S(H, G A)| \sum_{k=h}^{m} \frac{q^{k / 2}}{k}+\Phi(H) \sum_{k=h}^{m} \frac{q^{k / 2+l(k)}}{k}(l(k)+\operatorname{deg} H+1) \\
& \ll \operatorname{deg} H \Phi(H) \frac{q^{m / 2}}{m}+r \Phi(H) \frac{q^{5 m / 2-v(u)}}{m} \\
& \ll r|H| \frac{q^{5 m / 2-v(u)}}{m},
\end{aligned}
$$

d'où avec (2)

$$
\left|f(t)-\frac{S(H, G A)}{\Phi(H)} \sum_{k=1}^{m} \frac{q^{k}}{k} \Gamma_{k}^{\operatorname{sgn}(A)}(u)\right| \ll r|H| \frac{q^{5 m / 2-v(u)}}{m}+\frac{q^{h}}{h} .
$$


Proposition V.2. Soit $t=G / H+u$ appartenant à un arc de Farey de centre $G / H$ où $\operatorname{deg} H \leq 4 r+a_{i}$. Alors,

$$
\left|f_{i}(t)-\frac{S\left(H, G A_{i}\right)}{\Phi(H)} f_{i}^{*}(u)\right| \ll r|H| \frac{q^{5 m_{i} / 2-v(u)}}{m_{i}}+\frac{q^{4 r}}{r},
$$

où

$$
f_{i}^{*}(u)=\left\{\begin{array}{lr}
\tau_{m_{i}} & \text { si } v(u) \geq 2 m_{i}+a_{i}+2, \\
\tau_{j} & \text { si } v(u)=2(j+1)+a_{i} \\
& \text { avec } j \leq m_{i}-1, \\
\tau_{j-1}+\frac{q^{j}}{j} \sigma^{*}\left(\operatorname{sgn}(u) \alpha_{i}\right) & \text { si } v(u)=2 j+a_{i}+1, \\
\text { avec } j \leq m_{i}
\end{array}\right.
$$

et pour tout $t \in \mathbb{F}_{q}$,

$$
\sigma^{*}(t)=\sum_{y \in \mathbb{F}_{q}^{*}} \Psi\left(t y^{2}\right)
$$

Démonstration. On a

$$
f_{i}(t)=\sum_{\substack{P \in I^{*} \\ \operatorname{deg} P \leq m_{i}}} E\left(t A_{i} P^{2}\right)=\sum_{\alpha \in \mathbb{F}_{q}^{*}} \sum_{\substack{P \in I \\ \operatorname{deg} P \leq m_{i}}} E\left(t \alpha^{2} A_{i} P^{2}\right) .
$$

Compte tenu de l'inégalité (III.4) vérifiée par $n$, on a $4 r+a_{i}+1 \leq m_{i}$, on peut donc appliquer la proposition précédente avec $A=A_{i}, h=4 r+a_{i}+1$ et on a

$$
\left|f_{i}(t)-\sum_{k=1}^{m_{i}} \frac{q^{k}}{k} \sum_{\alpha \in \mathbb{F}_{q}^{*}} \frac{S\left(H, G \alpha^{2} A_{i}\right)}{\Phi(H)} \Gamma_{k}^{\alpha^{2} \alpha_{i}}(u)\right| \ll 4 r|H| \frac{q^{5 m_{i} / 2-v(u)}}{m_{i}}+\frac{q^{4 r}}{r} .
$$

L'application $Y \mapsto \alpha Y$ étant une bijection de $\mathcal{C}_{H}^{*} \operatorname{sur} \mathcal{C}_{H}^{*}$, on a alors

$$
\sum_{R \in \mathcal{C}_{H}^{*}} E\left(\frac{A_{i} G(\alpha R)^{2}}{H}\right)=\sum_{Y \in \mathcal{C}_{H}^{*}} E\left(\frac{A_{i} G Y^{2}}{H}\right)
$$

d'où

$$
S\left(H, G \alpha^{2} A_{i}\right)=S\left(H, G A_{i}\right)
$$

Posons

$$
\lambda_{k}(u)=\sum_{\alpha \in \mathbb{F}_{q}^{*}} \Gamma_{k}^{\alpha^{2} \alpha_{i}}(u)
$$

Alors

$$
\lambda_{k}(u)= \begin{cases}q-1 & \text { si } v(u)>2 k+a_{i}+1 \\ \sum_{\alpha \in \mathbb{F}_{q}^{*}} \Psi\left(\operatorname{sgn}(u) \alpha_{i} \alpha^{2}\right) & \text { si } v(u)=2 k+a_{i}+1 \\ 0 & \text { si } v(u) \leq 2 k+a_{i}\end{cases}
$$


Ainsi

$$
\left|f_{i}(t)-\frac{S\left(H, G A_{i}\right)}{\Phi(H)} \sum_{k=1}^{m_{i}} \frac{q^{k}}{k} \lambda_{k}(u)\right| \ll 4 r|H| \frac{q^{5 m_{i} / 2-v(u)}}{m_{i}}+\frac{q^{4 r}}{r} .
$$

Calculons maintenant $\sum_{k=1}^{m_{i}} \frac{q^{k}}{k} \lambda_{k}(u)$.

- Si $v(u)>a_{i}+2 m_{i}+1$, on a pour tout $k$ intervenant dans la somme ci-dessus, $v(u)>a_{i}+2 k+1, \lambda_{k}(u)=q-1$ et

$$
\sum_{k=1}^{m_{i}} \frac{q^{k}}{k} \lambda_{k}(u)=\tau_{m_{i}}
$$

- Si $v(u) \leq a_{i}+2 m_{i}+1$, il existe $j$ tel que $a_{i}+2 j+1 \leq v(u) \leq a_{i}+2(j+1)$, alors

$$
\sum_{k=1}^{m_{i}} \frac{q^{k}}{k} \lambda_{k}(u)=\sum_{k=1}^{j} \frac{q^{k}}{k} \lambda_{k}(u)+\sum_{k=j+1}^{m_{i}} \frac{q^{k}}{k} \lambda_{k}(u) .
$$

Or $\lambda_{k}(u)=0$ pour $k=j+1, \ldots, m_{i}$. Ainsi

$$
\sum_{k=1}^{m_{i}} \frac{q^{k}}{k} \lambda_{k}(u)=\sum_{k=1}^{j} \frac{q^{k}}{k} \lambda_{k}(u)
$$

Pour $v(u)>a_{i}+2 j+1$,

$$
\sum_{k=1}^{j} \frac{q^{k}}{k} \lambda_{k}(u)=\tau_{j}
$$

Pour $v(u)=a_{i}+2 j+1$,

$$
\sum_{k=1}^{j} \frac{q^{k}}{k} \lambda_{k}(u)=\sum_{k=1}^{j-1} \frac{q^{k}}{k} \lambda_{k}(u)+\frac{q^{j}}{j} \lambda_{j}(u)=\tau_{j-1}+\frac{q^{j}}{j} \sigma^{*}\left(\operatorname{sgn}(u) \alpha_{i}\right) .
$$

On a le résultat annoncé en posant

$$
f_{i}^{*}(u)=\sum_{k=1}^{m_{i}} \frac{q^{k}}{k} \lambda_{k}(u)
$$

VI. Majoration de $R_{2}^{-}(M)$. La majoration de $R_{2}^{-}(M)$ nécessite l'introduction d'une fonction auxiliaire $H$ que l'on va définir.

Soient $i<j$ des entiers pris parmi les $s$ premiers entiers. On désigne par $Y\left(A_{i}, A_{j}\right)$ le nombre de solutions $\left(Y_{i}, Y_{j}, Z_{i}, Z_{j}\right) \in\left(\mathbb{F}_{q}[X]\right)^{4}$ de l'equation

$$
A_{i} Y_{i}^{2}-A_{i} Z_{i}^{2}=A_{j} Z_{j}^{2}-A_{j} Y_{j}^{2}
$$

telles que $\operatorname{deg} Y_{i}, \operatorname{deg} Z_{i} \leq m_{i}$ et $\operatorname{deg} Y_{j}, \operatorname{deg} Z_{j} \leq m_{j}$. On pose

$$
N_{j}=a_{j}+m_{j}
$$


On pose aussi, pour $t \in \mathcal{P}, k \in\{i, j\}$,

$$
\begin{aligned}
h_{k}(t) & =\sum_{\operatorname{deg} Y \leq m_{k}} E\left(t A_{k} Y^{2}\right), \\
H(t) & =\left|h_{i}(t)\right|^{2}\left|h_{j}(t)\right|^{2} ;
\end{aligned}
$$

alors, d'après la proposition III.1,

$$
Y\left(A_{i}, A_{j}\right)=\int_{\mathcal{P}} H(t) d t .
$$

On fait une dissection de Farey de $\mathcal{P}$ à l'ordre $N_{j}$. On notera $\mathcal{V}_{G / K}$ l'arc de Farey $\mathcal{U}_{G / K, N_{j}}$. On appelle $\mathcal{P}_{1}$ la réunion des arcs de Farey $\mathcal{V}_{G / K}$ tels que $\operatorname{deg} K \leq m_{j}, \mathcal{P}_{2}$ la réunion des arcs restants. On pose

$$
\begin{aligned}
& Q_{1}=\int_{\mathcal{P}_{1}} H(t) d t, \\
& Q_{2}=\int_{\mathcal{P}_{2}} H(t) d t .
\end{aligned}
$$

Proposition VI.1. Soit $G / K$ une fraction de Farey telle que $\operatorname{deg} K \leq$ $m_{j}$. Posons

$$
J_{G / K}=\int_{\mathcal{V}_{G / K}} H(t) d t
$$

Alors

$$
J_{G / K} \geq 0
$$

$$
J_{G / K} \ll 2|K|^{-4}\left|C\left(K, G A_{i}\right)\right|^{2}\left|C\left(K, G A_{j}\right)\right|^{2} q^{2 n},
$$

où

$$
C(K, G)=\sum_{R \in \mathcal{C}_{K}} E\left(\frac{G R^{2}}{K}\right) .
$$

Démonstration. Puisque $H$ est une fonction positive, le (i) est évident. Supposons que $2 m_{j}+a_{j}>2 m_{i}+a_{i}$. Le corollaire VI.3 de [5] donne, pour $k=i, j$,

$$
\left|h_{k}(t)\right|^{2}=|K|^{-2}\left|h_{k}^{\prime}(u)\right|^{2}\left|C\left(K, G A_{k}\right)\right|^{2},
$$

avec

$$
h_{k}^{\prime}(u)= \begin{cases}q^{m_{k}+1} & \text { si } v\left(u A_{k}\right) \geq 2 m_{k}+2, \\ q^{k} & \text { si } v\left(u A_{k}\right)=2 k \leq 2 m_{k}, \\ q^{k} \sigma\left(\operatorname{sgn}\left(u A_{k}\right)\right) & \text { si } v(u)=2 k+1 \leq 2 m_{k}+1 .\end{cases}
$$


On a donc

$$
H(t)=|K|^{-4}\left|C\left(H, G A_{i}\right)\right|^{2}\left|C\left(H, G A_{j}\right)\right|^{2} H^{\prime}(u),
$$

où $H^{\prime}(u)=\left|h_{i}^{\prime}(u)\right|^{2}\left|h_{j}^{\prime}(u)\right|^{2}$. Comme $H^{\prime}(u)$ dépend uniquement de $v(u)$ et $\operatorname{sgn}(u)$, on pose $H^{\prime}(u)=\phi(v(u), \operatorname{sgn}(u))$. On a

$$
J_{G / K}=|K|^{-4}\left|C\left(K, G A_{i}\right)\right|^{2}\left|C\left(K, G A_{j}\right)\right|^{2} I,
$$

avec

$$
\begin{aligned}
I & =\int_{v(u) \geq 2 m_{j}+a_{j}+2} H^{\prime}(u) d u+\sum_{l=N_{j}+\operatorname{deg} K+1} \sum_{c \in \mathbb{F}_{q}^{*}} \phi(l, c) \int_{v(u)>l} d u \\
& =q^{2 m_{i}-a_{j}+3}+\sum_{l=N_{j}+\operatorname{deg} K+1}^{2 m_{j}+a_{j}+1} L_{l},
\end{aligned}
$$

où

$$
L_{l}=q^{-l} \sum_{c \in \mathbb{F}_{q}^{*}} \phi(l, c) .
$$

On déduit de la relation (5.9) de [16] que $|\sigma(\alpha)|^{2}=\left|\sum_{t \in \mathbb{F}_{q}} \Psi\left(\alpha t^{2}\right)\right|^{2}=q$ pour tout $\alpha \in \mathbb{F}_{q}^{*}$; alors $0 \leq \phi(l, c) \leq q^{2 l}$, donc $I \leq 2 q^{2 m_{j}+a_{j}+3}$, d'où le résultat.

Si $2 m_{i}+a_{i}>2 m_{j}+a_{j}$, on procède de façon identique.

Proposition VI.2. On a

$$
0 \leq Q_{1} \ll n q^{2 n}
$$

Démonstration. On a

$$
Q_{1}=\sum_{\operatorname{deg} K \leq m_{j}} \sum_{G \in \mathcal{C}_{K}^{*}} J_{G / K}
$$

donc

$$
Q_{1} \ll q^{2 n} \sum_{\operatorname{deg} K \leq m_{j}}|K|^{-4} \sum_{G \in \mathcal{C}_{K}^{*}}\left|C\left(K, G A_{i}\right)\right|^{2}\left|C\left(K, G A_{j}\right)\right|^{2} .
$$

D'après les propositions III.2 et III.3 de [5], on a

$$
\left|C\left(K, G A_{i}\right)\right| \leq\left|\left(K, A_{i}\right)\right|^{1 / 2}|K|^{1 / 2},
$$

d'où

$$
Q_{1} \ll q^{2 n} \sum_{\operatorname{deg} K \leq m_{j}}|K|^{-2} \Phi(K) .
$$

Comme $\sum_{\operatorname{deg} K \leq h}|K|^{-2} \Phi(K) \leq \sum_{\operatorname{deg} K \leq h}|K|^{-1}=h+1$, alors $Q_{1} \ll n q^{2 n}$.

Proposition VI.3. On a

$$
Q_{2} \ll q^{2 n} .
$$


Démonstration. Soit $t \in \mathcal{P}_{2}$. Alors $t$ s'écrit $t=u+G / K$ avec $m_{j}<$ $\operatorname{deg} K \leq N_{j}$ et $v(u) \geq N_{j}+\operatorname{deg} K+1 \geq 2 m_{j}+a_{j}+2$. On a donc

$$
h_{j}(t)=\sum_{\operatorname{deg} Y \leq m_{j}} E\left(\frac{G A_{j}}{K} Y^{2}\right) .
$$

- Si deg $K \leq m_{i}$, d'après le corollaire VI.3 de [5], on a

$$
h_{i}(t)=|K|^{-1} h_{i}^{\prime}(u) C\left(K, G A_{i}\right) .
$$

Alors,

$$
0 \leq\left|h_{i}(t)\right|^{2} \leq|K|^{-2}\left|h_{i}^{\prime}(u)\right|^{2}\left|C\left(K, G A_{i}\right)\right|^{2} \leq|K|^{-1}\left|\left(K, A_{i}\right)\right| q^{2 m_{i}+2} .
$$

Ainsi

$$
0 \leq\left|h_{i}(t)\right|^{2} \leq\left|A_{i}\right| q^{2 m_{i}-m_{j}+1} .
$$

- Si $\operatorname{deg} K>m_{i}$,

$$
h_{i}(t)=\sum_{\operatorname{deg} Y \leq m_{i}} E\left(\frac{G A_{i}}{K} Y^{2}\right) .
$$

Majorons donc

$$
S_{k}=\sum_{\operatorname{deg} Y \leq m_{k}} E\left(\frac{G A_{k}}{K} Y^{2}\right) .
$$

Comme il a été démontré dans la preuve de la proposition VII.2 de [5] on a

$$
\left|S_{k}\right|^{2} \leq q^{m_{k}+1} \#\left\{Y ; \operatorname{deg} Y<m_{k}, v\left(\left\{\frac{G A_{k}}{K} Y\right\}>m_{k}+1\right)\right\} .
$$

Si $\operatorname{deg}\left(K /\left(K, A_{k}\right)\right) \leq m_{k}+1$, on a

$$
\left|S_{k}\right|^{2}=q^{2 m_{k}+2}|K|^{-1}\left|\left(K, A_{k}\right)\right| \leq\left|A_{k}\right| q^{2 m_{k}-m_{j}+1} .
$$

Si $\operatorname{deg}\left(K /\left(K, A_{k}\right)\right)>m_{k}+1$, il vient

$$
\left|S_{k}\right|^{2} \leq|K| \leq q^{m_{j}+a_{j}}
$$

soit dans tous les cas

$$
\left|S_{k}\right|^{2} \leq\left|A_{j}\right| q^{2 m_{i}-m_{j}+1} \ll q^{n},
$$

d'où $|H(t)| \ll q^{n}$ et $\int_{\mathcal{P}_{1}}|H(t)| d t \ll q^{n}$.

Proposition VI.4. On a

$$
Y\left(A_{i}, A_{j}\right) \ll n q^{2 n} .
$$

Démonstration. On a $\int_{\mathcal{P}} H(t) d t=Y\left(A_{i}, A_{j}\right)$. Donc $Y\left(A_{i}, A_{j}\right)=Q_{1}+Q_{2}$. On conclut avec les relations (VI.8) et (VI.9).

Proposition VI.5. On a

$$
R_{2}^{-}(M) \ll \frac{q^{n(s-2)}}{n^{s}}\left(r n^{7} \log n q^{-r / 4}\right) .
$$


Démonstration. Soit $t \in \mathcal{M}_{2}^{\prime}$. Alors il existe une fraction de Farey $G / H$ et un indice $i$ tels que $t \in \mathcal{U}_{G / H}, 4 r<\operatorname{deg} H \leq 2 n-4 r$ et $\operatorname{deg} H_{i}^{*}>4 r$. Comme $v\left(u A_{i} P^{2}\right) \geq 2$, alors

$$
\begin{aligned}
f_{i}(t) & =\sum_{\substack{P \in I^{*} \\
\operatorname{deg} P \leq m_{i}}} E\left(t A_{i} P^{2}\right)=\sum_{\substack{P \in I^{*} \\
\operatorname{deg} P \leq m_{i}}} E\left(\frac{G A_{i}}{H_{i}} P^{2}\right) \\
& =\sum_{\substack{P \in I^{*} \\
\operatorname{deg} P \leq m_{i}}} E\left(\frac{G A_{i}^{*}}{H_{i}^{*}} P^{2}\right) .
\end{aligned}
$$

D'après la proposition VII.7 de [3], on a $\left|f_{i}(t)\right| \ll r n \log n q^{n-r / 4}$. Comme $F(t)=\prod_{j=1}^{s} f_{j}(t)$, alors

$$
|F(t)| \ll\left(\prod_{j=1, j \neq i}^{s}\left|f_{j}(t)\right|\right) r n \log n q^{n-r / 4} .
$$

Le théorème 1 nous donne $\left|f_{j}(t)\right| \ll q^{n} / n$. Ainsi

$$
\begin{aligned}
|F(t)| & \ll\left|f_{i_{1}}(t) f_{i_{2}}(t) f_{i_{3}}(t) f_{i_{4}}(t)\right| r n \log n q^{n-r / 4}\left(\frac{q^{n}}{n}\right)^{s-5} \\
& \ll\left|f_{i_{1}}(t) f_{i_{2}}(t) f_{i_{3}}(t) f_{i_{4}}(t)\right| n^{6-s} r \log n q^{n(s-4)} q^{-r / 4},
\end{aligned}
$$

où $i_{1}, i_{2}, i_{3}, i_{4}$ sont des indices distincts et différents de $i$. Alors

$$
\int_{\mathcal{M}_{2}^{\prime}}|F(t)| d t \ll n^{6-s} r \log n q^{n(s-4)-r / 4} \cdot L,
$$

où

$$
L=\int_{\mathcal{P}}\left|f_{i_{1}}(t) f_{i_{2}}(t) f_{i_{3}}(t) f_{i_{4}}(t)\right| d t
$$

L'inégalité de Cauchy-Schwarz donne

$$
L^{2} \leq L_{1,2} \cdot L_{3,4}, \quad \text { où } \quad L_{j, k}=\int_{\mathcal{P}}\left|f_{i_{j}}(t) f_{i_{k}}(t)\right|^{2} d t .
$$

Or

$$
\begin{aligned}
L_{j, k} & =\int_{\mathcal{P}} \sum_{\substack{P, P^{\prime}, Q, Q^{\prime} \in I^{*} \\
\operatorname{deg} P, \operatorname{deg} Q \leq m_{i_{j}} ; \operatorname{deg} P^{\prime}, \operatorname{deg} Q^{\prime} \leq m_{i_{k}}}} E\left(t\left(A_{i_{j}}\left(P^{2}-Q^{2}\right)+A_{i_{k}}\left(P^{\prime 2}-Q^{\prime 2}\right)\right),\right. \\
& =Y^{*}\left(A_{i_{j}}, A_{i_{k}}\right),
\end{aligned}
$$

où $Y^{*}\left(A_{i_{j}}, A_{i_{k}}\right)$ est le nombre de solutions $\left(P, P^{\prime}, Q, Q^{\prime}\right) \in\left(I^{*}\right)^{4}$ de l'equation

$$
A_{i_{j}} Y_{i_{j}}^{2}-A_{i_{j}} Z_{i_{j}}^{2}=A_{i_{k}} Z_{i_{k}}^{2}-A_{i_{k}} Y_{i_{k}}^{2}
$$

telles que $\operatorname{deg} P, \operatorname{deg} Q \leq m_{i_{j}}$ et $\operatorname{deg} P^{\prime}, \operatorname{deg} Q^{\prime} \leq m_{i_{k}}$. De façon évidente on a $Y^{*}\left(A_{i_{j}}, A_{i_{k}}\right) \leq Y\left(A_{i_{j}}, A_{i_{k}}\right)$. On conclut à l'aide de la proposition VI.4. 
VII. Approximation de $R(M)$. Nous reprenons les hypothèses du paragraphe IV. Notons aussi que pour tout $B \subset\{1, \ldots, s\}$, on a

$$
\prod_{i \in B} \tau_{m_{i}}=\prod_{i \in B} \tau_{m_{i}-1}+\sum_{\substack{I \cup J=B \\ I \cap J=\emptyset, J \neq \emptyset}} \prod_{i \in I} \tau_{m_{i}-1} \prod_{i \in J} \frac{q^{m_{i}}}{m_{i}}(q-1)^{\# J} .
$$

Proposition VII.1. Soit $\varepsilon$ un réel positif. Soit $G / H$ le centre d'un arc de Farey. Alors :

(i) Si $G / H$ est le centre d'un arc majeur,

$$
\begin{aligned}
\mid I_{G / H}(M)-\frac{T(H, G)}{\Phi(H)^{s}} E(-M & \left.\frac{G}{H}\right)\left(J_{s}(M)+j_{s}(H, M)\right) \mid \\
& \ll|H|^{(\varepsilon-1 / 2)(s-1)-1} q^{n(s-5 / 2)} q^{8 r},
\end{aligned}
$$

où

(VII.2) $\quad J_{s}(M)$

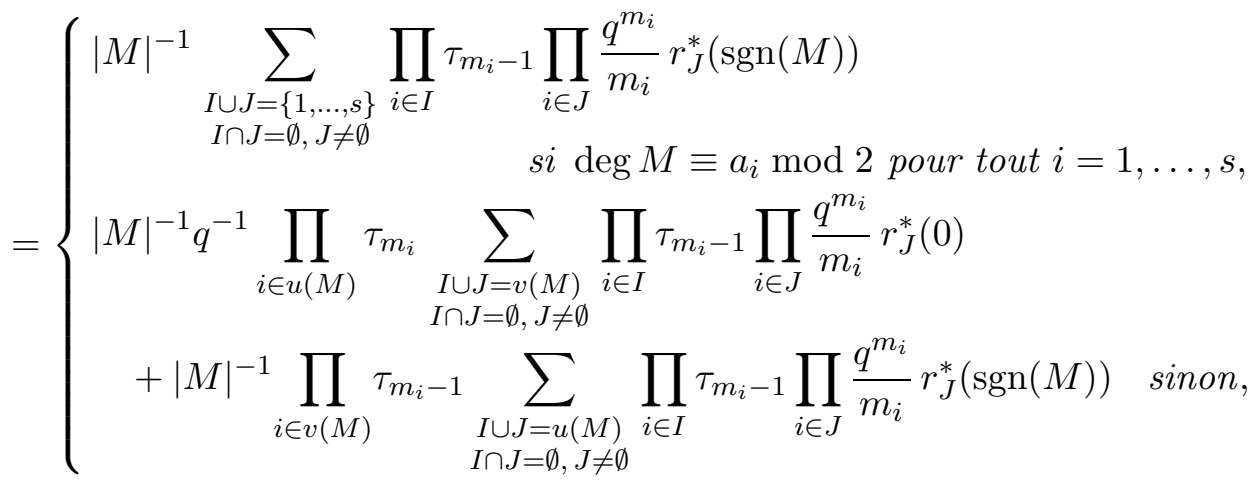

et

(VII.3)

$$
\left|j_{s}(H, M)\right| \leq \begin{cases}0 & \text { si } \operatorname{deg} M=2 n \\ & \text { ou } \operatorname{deg} M=2 n-1 \text { et } \operatorname{deg} H<4 r, \\ |M|^{-1} \prod_{i=1}^{s} \tau_{m_{i}} & \text { si } \operatorname{deg} M=2 n-1 \text { et } \operatorname{deg} H=4 r,\end{cases}
$$

la constante intervenant dans le symbole $\ll$ ne dépendant que de $q, s$, $\varepsilon$ et $A_{1}, \ldots, A_{s}$.

(ii) Si $G / H$ est le centre d'un arc médian,

$$
\left|I_{G / H}(M)\right| \ll \frac{|T(H, G)|}{\Phi(H)^{s}}|H|^{-1} \frac{q^{n(s-2)}}{n^{s}} q^{4 r} .
$$

Démonstration. Soit $t=G / H+u$ appartenant à un arc de Farey $\mathcal{U}_{G / H}$ où $\operatorname{deg} H \leq 4 r+a_{1}$. Alors, avec (V.5),

$$
F(t)=\prod_{i=1}^{s} \frac{S\left(H, G A_{i}\right)}{\Phi(H)} F^{*}(u)+e(t),
$$


où

$$
F^{*}(u)=\prod_{i=1}^{s} f_{i}^{*}(u), \quad e(t)=\sum_{\substack{I \cup J=\{1, \ldots, s\} \\ J \neq \emptyset}} \prod_{i \in I} \frac{S\left(H, G A_{i}\right)}{\Phi(H)} f_{i}^{*}(u) \cdot \prod_{j \in J} e_{j}(t)
$$

et

$$
e_{i}(t) \ll r|H| \frac{q^{5 n / 2-v(u)}}{n}+\frac{q^{4 r}}{r} .
$$

Or, d'après la proposition V.4 de [4] on a

$$
\Phi(H) \gg|H|(\log \operatorname{deg} H)^{-1} .
$$

Avec la relation (IV.9), on déduit que

$$
\frac{\left|S\left(H, G A_{i}\right)\right|}{\Phi(H)} \ll(\log \operatorname{deg} H)|H|^{\varepsilon-1 / 2},
$$

la constante intervenant dans $\ll$ ne dépendant que de $q, \varepsilon$ et $A_{i}$. D'où

$$
\begin{aligned}
e(t) & \ll \sum_{\substack{I \cup J=\{1, \ldots, s\} \\
J \neq \emptyset}}\left(r|H|^{\varepsilon-1 / 2} \frac{q^{n}}{n}\right)^{\# I}\left[\left(r|H| \frac{q^{5 / 2} n-v(u)}{n}\right)^{\# J}+\left(\frac{q^{4 r}}{r}\right)^{\# J}\right] \\
& \ll \sum_{i=0}^{s-1}\left(r|H|^{\varepsilon-1 / 2} \frac{q^{n}}{n}\right)^{i}\left[\left(r|H| \frac{q^{5 / 2} n-v(u)}{n}\right)^{s-i}+\left(\frac{q^{4 r}}{r}\right)^{s-i}\right] \\
& \ll\left(r|H|^{\varepsilon-1 / 2} \frac{q^{n}}{n}\right)^{s-1}\left[r|H| \frac{q^{5 / 2} n-v(u)}{n}+\frac{q^{4 r}}{r}\right],
\end{aligned}
$$

où les constantes intervenant dans « ne dépendent que de $q, s, \varepsilon$ et $A_{1}, \ldots$ $\ldots, A_{s}$. Posons

$$
K_{s}(M)=\int_{v(u)>N+\operatorname{deg} H} F^{*}(u) E(-M u) d u .
$$

Alors

$$
\begin{aligned}
& \left|I_{G / H}(M)-\frac{T(H, G)}{\Phi(H)^{s}} E\left(-M \frac{G}{H}\right) K_{s}(M)\right| \\
& \ll r^{s}|H|^{(\varepsilon-1 / 2)(s-1)+1} \frac{q^{n(s+3 / 2)}}{n^{s}} \int_{v(u)>N+\operatorname{deg} H} q^{-v(u)} d u \\
& \quad+r^{s-2}|H|^{(\varepsilon-1 / 2)(s-1)} \frac{q^{(s-1) n}}{n^{s-1}} \cdot \frac{q^{4 r}}{r} q^{-N-\operatorname{deg} H} \\
& \ll r^{s}|H|^{(\varepsilon-1 / 2)(s-1)-1} \frac{q^{n(s-5 / 2)}}{n^{s}} q^{8 r},
\end{aligned}
$$

les constantes intervenant dans $\ll$ ne dépendant que de $q, s, \varepsilon$ et $A_{1}, \ldots, A_{s}$. 
(i) Supposons que $G / H$ soit le centre d'un arc majeur. Comme $F^{*}$ ne dépend que de $v(u)$ et de $\operatorname{sgn}(u)$, on pose alors

$$
F^{*}(u)=\phi(\operatorname{sgn}(u), v(u)) .
$$

On remarque que $F^{*}(u)$ est constante sur l'ensemble $\{u \in \mathcal{P} ; v(u)>1+\lambda\}$, où

$$
\lambda=\max \left\{2 m_{1}+a_{1}, \ldots, 2 m_{s}+a_{s}\right\} .
$$

D'après (V.6),

$$
\begin{aligned}
K_{s}(M)= & \prod_{i=1}^{s} \tau_{m_{i}} \int_{v(u)>1+\lambda} E(-M u) d u \\
& +\sum_{j=1+N+\operatorname{deg}}^{1+\lambda} \sum_{c \in \mathbb{F}_{q}^{*}} \phi(j, c) E\left(-M c X^{-j}\right) \int_{v(u)>j} E(-M u) d u,
\end{aligned}
$$

d'où avec (III.1),

$$
\begin{aligned}
K_{s}(M) & =q^{-1-\lambda} \prod_{i=1}^{s} \tau_{m_{i}}+\sum_{j=1+\max (N+\operatorname{deg} H, \operatorname{deg} M)}^{1+\lambda} q^{-j} \sum_{c \in \mathbb{F}_{q}^{*}} \phi(j, c) E\left(-M c X^{-j}\right) \\
& =J_{s}(M)+j_{s}(H, M),
\end{aligned}
$$

où

$$
\begin{gathered}
J_{s}(M)=q^{-1-\lambda} \prod_{i=1}^{s} \tau_{m_{i}}+\sum_{j=1+\operatorname{deg} M}^{1+\lambda} q^{-j} \sum_{c \in \mathbb{F}_{q}^{*}} \phi(j, c) E\left(-M c X^{-j}\right), \\
j_{s}(H, M)=-\sum_{j=1+\operatorname{deg} M}^{\max (N+\operatorname{deg} H, \operatorname{deg} M)} q^{-j} \sum_{c \in \mathbb{F}_{q}^{*}} \phi(j, c) E\left(-M c X^{-j}\right) .
\end{gathered}
$$

Calculons d'abord $J_{s}(M)$.

(a) Si $\lambda=\operatorname{deg} M$ alors pour tout $i \in\{1, \ldots, s\}, \operatorname{deg} M=2 m_{i}+a_{i}$ et $a_{i}$ est de même parité que $\operatorname{deg} M$. On applique la définition du caractère $E$. La relation (V.6) nous donne

$$
J_{s}(M)=q^{-1-\lambda} \prod_{i=1}^{s} \tau_{m_{i}}+q^{-1-\lambda} \sum_{c \in \mathbb{F}_{q}^{*}} \Psi(-c \operatorname{sgn}(M)) \prod_{i=1}^{s}\left(\tau_{m_{i}-1}+\frac{q^{m_{i}}}{m_{i}} \sigma\left(\alpha_{i} c\right)\right),
$$

d'où

$$
\begin{aligned}
J_{s}(M)= & q^{-1-\lambda}\left(\left(\prod_{i=1}^{s} \tau_{m_{i}}-\prod_{i=1}^{s} \tau_{m_{i}-1}\right)\right. \\
& \left.+\sum_{\substack{I \cup J=\{1, \ldots, s\} \\
I \cap J=\emptyset, J \neq \emptyset}} \prod_{\substack{i \in I \\
\tau_{m_{i}-1}}} \prod_{i \in J} \frac{q^{m_{i}}}{m_{i}}\left(q r_{J}^{*}(\operatorname{sgn}(M))-(q-1)^{\# J}\right)\right),
\end{aligned}
$$


donc

$$
J_{s}(M)=q^{-\lambda} \sum_{\substack{I \cup J=\{1, \ldots, s\} \\ I \cap J=\emptyset, J \neq \emptyset}} \prod_{i \in I} \tau_{m_{i}-1} \prod_{i \in J} \frac{q^{m_{i}}}{m_{i}} r_{J}^{*}(\operatorname{sgn}(M)),
$$

ce qui est l'égalité cherchée.

(b) Si $\lambda>\operatorname{deg} M$ alors $\operatorname{deg} M=\lambda-1$.

- Si $\operatorname{deg} M=2 m_{i}+a_{i}$ (i.e. $i \in u(M)$ ), pour $v(u)=1+\lambda=2 m_{i}+a_{i}+2$,

$$
f_{i}^{*}(u)=\tau_{m_{i}}
$$

et pour $v(u)=\lambda=2 m_{i}+a_{i}+1$,

$$
f_{i}^{*}(u)=\tau_{m_{i}-1}+\frac{q^{m_{i}}}{m_{i}} \sigma\left(\alpha_{i} c\right) .
$$

- Si $\operatorname{deg} M=2 m_{i}+a_{i}-1$ (i.e. $i \in v(M)$ ), pour $v(u)=1+\lambda=2 m_{i}+a_{i}+1$,

$$
f_{i}^{*}(u)=\tau_{m_{i}-1}+\frac{q^{m_{i}}}{m_{i}} \sigma\left(\alpha_{i} c\right)
$$

et pour $v(u)=\lambda=2 m_{i}+a_{i}$,

$$
f_{i}^{*}(u)=\tau_{m_{i}}
$$

D'où

$$
\begin{aligned}
J_{s}(M)= & q^{-1-\lambda} \prod_{i=1}^{s} \tau_{m_{i}}+q^{-1-\lambda} \prod_{i \in u(M)} \tau_{m_{i}} \sum_{c \in \mathbb{F}_{q}^{*}} \prod_{i \in v(M)}\left(\tau_{m_{i}-1}+\frac{q^{m_{i}}}{m_{i}} \sigma\left(\alpha_{i} c\right)\right) \\
& +q^{-\lambda} \prod_{i \in v(M)} \tau_{m_{i}-1} \sum_{c \in \mathbb{F}_{q}^{*}} \prod_{i \in u(M)}\left(\tau_{m_{i}-1}+\frac{q^{m_{i}}}{m_{i}} \sigma\left(\alpha_{i} c\right)\right) \\
& \times \Psi(-c \operatorname{sgn}(M)),
\end{aligned}
$$

donc

$$
\begin{aligned}
J_{s}(M)= & q^{-1-\lambda} \prod_{i=1}^{s} \tau_{m_{i}}+q^{-1-\lambda} \prod_{i \in u(M)} \tau_{m_{i}}\left((q-1) \prod_{i \in v(M)} \tau_{m_{i}-1}\right. \\
& \left.+\sum_{\substack{I \cup J=v(M) \\
I \cap J=\emptyset, J \neq \emptyset}} \prod_{i \in I} \tau_{m_{i}-1} \prod_{i \in J} \frac{q^{m_{i}}}{m_{i}} q r_{J}^{*}(0)-\prod_{i \in v(M)} \tau_{m_{i}}+\prod_{i \in v(M)} \tau_{m_{i}-1}\right) \\
& +q^{-\lambda} \prod_{i \in v(M)} \tau_{m_{i}-1}\left(-\prod_{i \in u(M)} \tau_{m_{i}-1}+\sum_{\substack{I \cup J=u(M) \\
I \cap J=\emptyset, J \neq \emptyset}} \prod_{i \in I} \tau_{m_{i}-1}\right. \\
& \left.\times \prod_{i \in J} \frac{q^{m_{i}}}{m_{i}} q r_{J}^{*}(\operatorname{sgn}(M))-\prod_{i \in u(M)} \tau_{m_{i}}+\prod_{\substack{i \in u(M) \\
i \in m_{i}-1}}\right)
\end{aligned}
$$


par suite

$$
\begin{aligned}
J_{s}(M)= & q^{-\lambda} \prod_{i \in u(M)} \tau_{m_{i}} \sum_{\substack{I \cup J=v(M) \\
I \cap J=\emptyset, J \neq \emptyset}} \prod_{i \in I} \tau_{m_{i}-1} \prod_{i \in J} \frac{q^{m_{i}}}{m_{i}} r_{J}^{*}(0)+q^{1-\lambda} \prod_{i \in v(M)} \tau_{m_{i}-1} \\
& \times \sum_{\substack{I \cup J=u(M) \\
I \cap J=\emptyset, J \neq \emptyset}} \prod_{i \in I} \tau_{m_{i}-1} \prod_{i \in J} \frac{q^{m_{i}}}{m_{i}} r_{J}^{*}(\operatorname{sgn}(M)) .
\end{aligned}
$$

La majoration (VII.3) se déduit de (V.6).

(ii) Supposons que $G / H$ soit le centre d'un arc médian. Puisque $v(u) \geq$ $2 n+2 \geq \operatorname{deg} M+2$, alors

$$
\left|K_{s}(M)\right| \ll \frac{q^{n s}}{n^{s}} q^{-N-\operatorname{deg} H} \ll|H|^{-1} \frac{q^{n(s-2)}}{n^{s}} q^{4 r} .
$$

D'où la majoration de $I_{G / H}(M)$.

Corollaire 3. Pour $s \geq 5$ et $\varepsilon>0$, on a la relation

$$
\begin{aligned}
\mid R^{+}(M)-J_{s}(M) \sum_{\substack{H \in \mathbb{M} \\
\operatorname{deg} H \leq 4 r}} A_{s}(H, M)- & \sum_{\substack{H \in \mathbb{M} \\
\operatorname{deg} H \leq 4 r}} j_{s}(H, M) A_{s}(H, M) \mid \\
& \ll q^{n(s-5 / 2)} q^{4 r[(\varepsilon-1 / 2)(s-1)+3]}
\end{aligned}
$$

la constante dans $\ll$ ne dépendant que de $q, s, \varepsilon$ et $A_{1}, \ldots, A_{s}$.

Proposition VII.2. Pour tout réel $\varepsilon \in] 0,1 / 2[$ on a

$$
R_{1}^{-}(M) \ll \frac{q^{n(s-2)}}{n^{s}} q^{4 r(\varepsilon+2-s / 2)},
$$

la constante dans $\ll$ ne dépendant que de $q, s$, $\varepsilon$ et $A_{1}, \ldots, A_{s}$.

Démonstration. Si $G / H$ est le centre d'un arc médian alors $4 r<\operatorname{deg} H$ $\leq 4 r+a_{1}$. D'après la relation (VII.4),

$$
R_{1}^{-}(M) \ll \frac{q^{n(s-2)}}{n^{s}} q^{4 r} \sum_{\substack{H \in \mathbb{M} \\ 4 r<\operatorname{deg} H \leq 4 r+a_{1}}}|H|^{-1} \Phi(H)^{-s} \sum_{G \in \mathcal{C}_{H}^{*}}|T(H, G)| .
$$

D'après la majoration $(* *)$ de la démonstration de la proposition IV.9 on a

$$
\begin{aligned}
R_{1}^{-}(M) & \ll \frac{q^{n(s-2)}}{n^{s}} q^{4 r} \sum_{\operatorname{deg} H>4 r}|H|^{\varepsilon-s / 2} \\
& \ll \frac{q^{n(s-2)}}{n^{s}} q^{4 r} \sum_{j=4 r+1}^{\infty} q^{j(\varepsilon+1-s / 2)} \ll \frac{q^{n(s-2)}}{n^{s}} q^{(\varepsilon+2-s / 2) 4 r},
\end{aligned}
$$

où les constantes intervenant dans le symbole $\ll$ ne dépendent que de $q, s$, $\varepsilon$ et $A_{1}, \ldots, A_{s}$. 
Lemme 3. (i) Si $q=7$ ou $q>9$, pour tout polynôme $M$ de $\mathbb{F}_{q}[X]$, on a (VII.7)

$$
\frac{q^{n(s-2)}}{n^{s}} \ll J_{s}(M) \ll \frac{q^{n(s-2)}}{n^{s}} .
$$

Si $q=5$ ou $q=9$, et si au moins quatre polynômes $A_{i_{1}}, \ldots, A_{i_{4}}$ parmi $A_{1}, \ldots, A_{s}$ ont des degrés de même parité, le même résultat reste valable pour les polynômes $M$ de degré $\operatorname{deg} M \equiv \operatorname{deg} A_{i_{1}} \bmod 2$.

(ii) Pour $\operatorname{deg} M=2 n-1$ et $\operatorname{deg} H=4 r$ on $a$

$$
\frac{q^{n(s-2)}}{n^{s}} \ll j_{s}(H, M) \ll \frac{q^{n(s-2)}}{n^{s}} .
$$

Démonstration. (i) D'après la relation (IV.12) pour tout $b \in \mathbb{F}_{q}$, on a

$$
q r_{J}^{*}(b) \leq(q-1)^{\# J}+(q-1)(1+\sqrt{q})^{\# J} \leq q(q-1)^{\# J} .
$$

- On suppose que $\operatorname{deg} M \equiv a_{i} \bmod 2$ pour tout $i=1, \ldots, s$. La relation (VII.2) nous donne donc

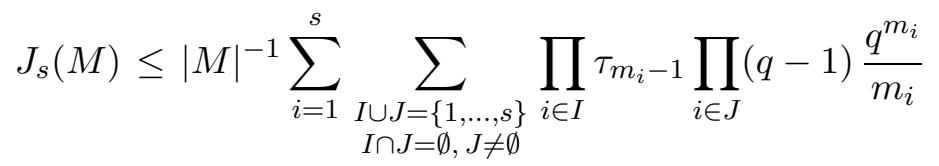

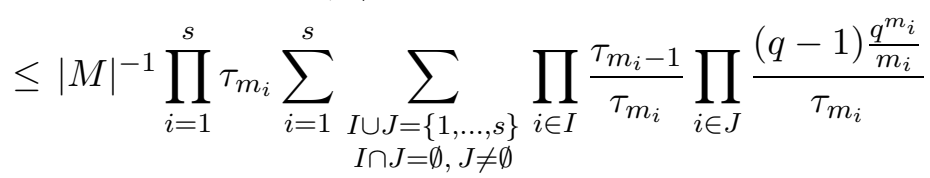

$$
\begin{aligned}
& \ll q^{-2 n}\left(\frac{q^{n}}{n}\right)^{s} .
\end{aligned}
$$

La majoration s'en déduit.

Comme $s \geq 5$, l'ensemble d'indices $u(M)$ contient un ensemble $J_{0}$ a cinq éléments et $r_{J_{0}}^{*}(\operatorname{sgn}(M)) \geq 1$ d'après la relation (IV.14). Or $\tau_{j} \geq(q-1) q^{j} / j$, alors

$$
J_{s}(M) \geq|M|^{-1} \prod_{i \in\{1, \ldots, s\} \backslash J_{0}} \tau_{m_{i}-1} \prod_{i \in J_{0}} \frac{q^{m_{i}}}{m_{i}} \gg q^{-2 n}\left(\frac{q^{n}}{n}\right)^{s} .
$$

- On suppose qu'il existe $j \in\{1, \ldots, s\}$ tel que $\operatorname{deg} M \not \equiv a_{j} \bmod 2$. Alors $\# v(M) \geq 1$.

Si $q=7$ ou $q>9$, et si $\# v(M) \geq 3$, il existe $J_{1} \subset v(M)$ à trois éléments. D'après la relation (IV.15), $r_{J_{1}}^{*}(0) \geq 1$. Dans ce cas,

$$
J_{s}(M) \geq q^{-1}|M|^{-1} \prod_{i \in u(M)} \tau_{m_{i}} \prod_{i \in v(M) \backslash J_{1}} \tau_{m_{i}-1} \prod_{i \in J_{1}} \frac{q^{m_{i}}}{m_{i}} \gg q^{-2 n}\left(\frac{q^{n}}{n}\right)^{s} .
$$

Si $q=7$ ou $q>9$ et si $\# v(M) \leq 2$, alors $\# u(M) \geq s-2 \geq 3$. Il existe $J_{2} \subset u(M)$ à trois éléments et d'après la relation (IV.15), $r_{J_{2}}^{*}(\operatorname{sgn}(M)) \geq 1$. 
Dans ce cas,

$$
J_{s}(M) \geq|M|^{-1} \prod_{i \in v(M)} \tau_{m_{i}-1} \prod_{i \in u(M) \backslash J_{2}} \tau_{m_{i}-1} \prod_{i \in J_{2}} \frac{q^{m_{i}}}{m_{i}} \gg q^{-2 n}\left(\frac{q^{n}}{n}\right)^{s} .
$$

Si $q=5$ ou $q=9$, on a $\# u(M) \geq 4$, il existe donc $I \subset u(M)$ à quatre éléments tel que $r_{I}^{*}(\operatorname{sgn}(M)) \geq 1$. Alors

$$
J_{s}(M) \geq|M|^{-1} \prod_{i \in v(M)} \tau_{m_{i}} \prod_{i \in u(M) \backslash I} \tau_{m_{i}-1} \prod_{i \in I} \frac{q^{m_{i}}}{m_{i}} \gg q^{-2 n}\left(\frac{q^{n}}{n}\right)^{s} .
$$

La majoration se démontre comme la majoration précédente.

(ii) Les relations (VII.8) sont évidentes.

Proposition VII.3. Pour $s \geq 5$, on a

$$
\left|R_{s}(M)-J_{s}(M) \mathfrak{S}_{s}(M)\right| \ll \frac{q^{n(s-2)}}{n^{s}}\left(r n^{7} \log n q^{-r / 4}\right) .
$$

Démonstration. On a

$$
\begin{aligned}
& \left|R_{s}(M)-J_{s}(M) \mathfrak{S}_{s}(M)\right| \\
& \leq\left|R^{+}(M)-J_{s}(M) \sum_{\substack{H \in \mathbb{M} \\
\operatorname{deg} H \leq 4 r}} A_{s}(H, M)-\sum_{\substack{H \in \mathbb{M} \\
\operatorname{deg} H \leq 4 r}} j_{s}(H, M) A_{s}(H, M)\right| \\
& \quad+\left|J_{s}(M)\right| \sum_{\substack{H \in \mathbb{M} \\
\operatorname{deg} H>4 r}}\left|A_{s}(H, M)\right|+\sum_{\substack{H \in \mathbb{M} \\
\operatorname{deg} H=4 r}}\left|j_{s}(H, M)\right|\left|A_{s}(H, M)\right| \\
& \quad+\left|R_{1}^{-}(M)\right|+\left|R_{2}^{-}(M)\right| .
\end{aligned}
$$

Soit $\varepsilon \in] 0,1 / 4[$. Les relations (IV.20), (VI.11), (VII.5)-(VII.8) nous donnent

$$
\begin{aligned}
\left|R_{s}(M)-J_{s}(M) \mathfrak{S}_{s}(M)\right| \ll & q^{n(s-5 / 2)} q^{4 r[(\varepsilon-1 / 2)(s-1)+3]}+\frac{q^{n(s-2)}}{n^{s}} q^{4 r(\varepsilon+2-s / 2)} \\
& +\frac{q^{n(s-2)}}{n^{s}}\left(r n^{7} \log n q^{-r / 4}\right) \\
\ll & \frac{q^{n(s-2)}}{n^{s}}\left(r n^{7} \log n q^{-r / 4}\right),
\end{aligned}
$$

ce qui est le résultat annoncé.

Corollaire 4. Sous les mêmes hypothèses du lemme 3 , on a

$$
R_{s}(M) \gg(\operatorname{deg} M)^{-s}|M|^{s / 2-1} .
$$

Démonstration. D'après la proposition précédente on a

$$
R_{s}(M) \gg J_{s}(M) \mathfrak{S}_{s}(M)-\frac{q^{n(s-2)}}{n^{s}}\left(r n^{7} \log n q^{-r / 4}\right) .
$$


D'après les relations (IV.21) et (VII.7) on a $J_{s}(M) \mathfrak{S}_{s}(M)>0$ et

$$
J_{s}(M) \mathfrak{S}_{s}(M) \gg(\operatorname{deg} M)^{-s} q^{\operatorname{deg} M(s / 2-1)},
$$

d'où le résultat annoncé.

\section{Références}

[1] R. Ayoub, An Introduction to the Analytic Theory of Numbers, Math. Surveys Monogr. 10, Amer. Math. Soc., Providence, RI, 1963.

[2] M. Car, Sommes de carrés de polynômes irréductibles dans $\mathbb{F}_{q}[X]$, Acta Arith. 44 (1984), 307-321.

[3] - Sommes de carrés et d'irréductibles dans $\mathbb{F}_{q}[X]$, Ann. Fac. Sci. Toulouse 3 (1981), 129-166.

[4] - Sommes de puissances et d'irréductibles dans $\mathbb{F}_{q}[X]$, Acta Arith. 44 (1984), $7-34$

[5] —, Quadratic forms on $\mathbb{F}_{q}[T]$, J. Number Theory 61 (1996), 145-180.

[6] L. Carlitz, On the representation of a polynomial on a Galois field as the sum of an even number of squares, Trans. Amer. Math. Soc. 35 (1933), 397-410.

[7] - , On the representation of a polynomial on a Galois field as the sum of an odd number of squares, Duke Math. J. 1 (1935), 298-315.

[8] -, Sums of squares of polynomials, ibid. 3 (1937), 1-7.

[9] —, The singular series for sums of squares of polynomials, ibid. 14 (1947), 11051120.

[10] -, A note on sums of three squares in $G F[q, x]$, Math. Mag. 48 (1975), 109-110.

[11] E. Cohen, Sums of an even number of squares in $G F\left[p^{n}, x\right], I$, Duke Math. J. 14 (1947), 251-267.

[12] -, Sums of an even number of squares in $G F\left[p^{n}, x\right]$, II, ibid. 14 (1947), 543-557.

[13] G. Effinger and D. Hayes, Additive Number Theory of Polynomials over a Finite Field, Oxford Univ. Press, Oxford, 1991.

[14] D. R. Hayes, The expression of a polynomial as a sum of three irreducibles, Acta Arith. 11 (1966), 461-488.

[15] C. N. Hsu, The distribution of irreducible polynomials in $\mathbb{F}_{q}[T]$, J. Number Theory 61 (1996), 85-96.

[16] R. Lidl and H. Niederreiter, Introduction to Finite Fields and their Applications, Cambridge Univ. Press, Cambridge, 1994.

[17] G. Rhin, Répartition modulo 1 dans un corps de séries formelles sur un corps fini, Dissertationes Math. 95 (1972).

[18] W. Webb, Waring's problem in $G F[q, x]$, Acta Arith. 22 (1972), 207-220.

Département de Mathématiques

Faculté des Sciences de Tunis

1060 Tunis, Tunisie

E-mail: anis.rezgui@fsb.rnu.tn 